\title{
Erratum to: Buckwheat flour inclusion in Chinese steamed bread: potential reduction in glycemic response and effects on dough quality
}

\author{
Wenjun Liu ${ }^{1} \cdot$ Margaret Brennan $^{1} \cdot$ Luca Serventi $^{1} \cdot$ Charles Brennan $^{1}$
}

Published online: 9 November 2016

(C) Springer-Verlag Berlin Heidelberg 2016

\section{Erratum to: Eur Food Res Technol DOI 10.1007/s00217-016-2786-x}

In the original publication, all the figures and tables presented in the paper are inaccurate as they are derived from another experiment. The correct figures and tables are given here (Figs. 1, 2; Tables 1, 2, 3, 4, 5, 6).

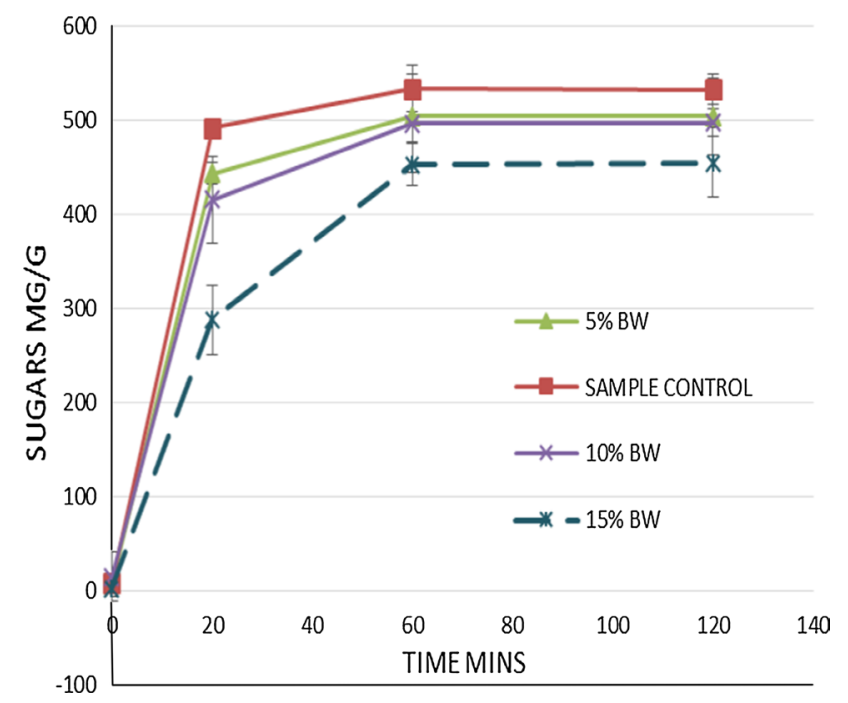

Fig. 1 Reducing sugar released during in vitro digestion

The online version of the original article can be found under doi:10.1007/s00217-016-2786-x.

Margaret Brennan

Margaret.Brennan@lincoln.ac.nz

Department of Wine, Food and Molecular Biosciences,

Faculty of Agriculture and Life Sciences, Lincoln University,

Lincoln, Christchurch 7647, New Zealand

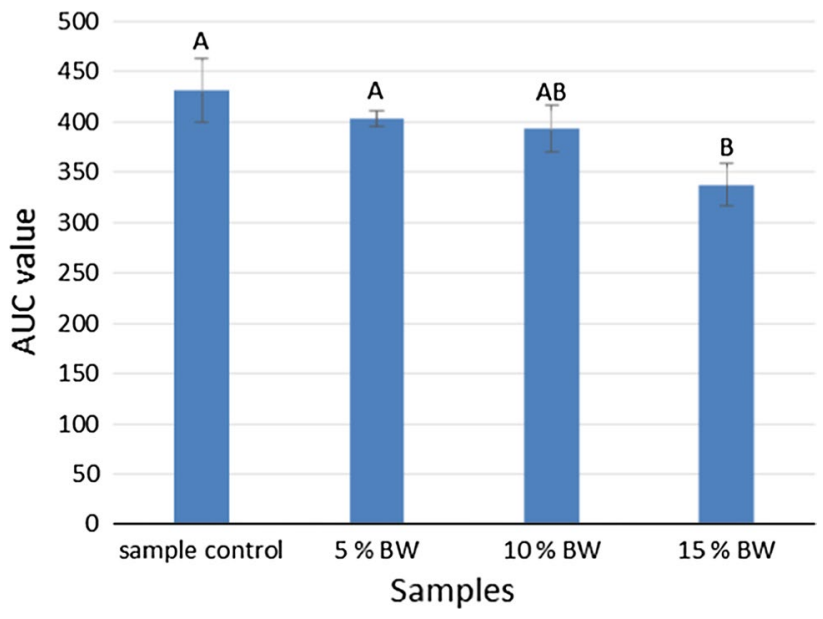

Fig. 2 Standardized area under curve values from predictive in vitro glycemic digestion

Table 1 Nutrition information (from Champion Flour Milling Ltd and Ceres Organics Ltd)

\begin{tabular}{lll}
\hline Per $100 \mathrm{~g}$ & Wheat flour $(\mathrm{g})$ & Buckwheat flour $(\mathrm{g})$ \\
\hline Protein & 11.0 & 13.3 \\
Fat, total & 1.4 & 3.4 \\
$\quad$ Saturated & $<1$ & 0.7 \\
Carbohydrate & 77.3 & 71.5 \\
$\quad$ Sugars & $<1$ & 3.0 \\
Dietary fibre & 3.1 & 10.0 \\
Sodium & 0.005 & 0.011 \\
\hline
\end{tabular}


Table 2 Rheological properties of dough incorporated with buckwheat flour

\begin{tabular}{lllllll}
\hline Sample & WA $(\%)$ & Stability $(\min )$ & Development time (min) & Softening (FU) & Departure time (min) & MTI (FU) \\
\hline Wheat flour & $63.67 \pm 0.11^{\mathrm{C}}$ & $11.17 \pm 0.47^{\mathrm{A}}$ & $2.73 \pm 0.06^{\mathrm{C}}$ & $41.46 \pm 4.11^{\mathrm{C}}$ & $12.72 \pm 0.85^{\mathrm{A}}$ & $10.51 \pm 1.10^{\mathrm{B}}$ \\
Wheat flour + 5\% BW & $64.32 \pm 0.02^{\mathrm{B}}$ & $10.07 \pm 0.11^{\mathrm{B}}$ & $5.63 \pm 0.58^{\mathrm{B}}$ & $66.83 \pm 0.21^{\mathrm{A}}$ & $11.67 \pm 0.21^{\mathrm{A}}$ & $30.01 \pm 6.00^{\mathrm{A}}$ \\
Wheat flour + 10\% BW & $64.31 \pm 0.11^{\mathrm{B}}$ & $10.17 \pm 0.21^{\mathrm{B}}$ & $6.76 \pm 0.15^{\mathrm{A}}$ & $59.23 \pm 1.62^{\mathrm{AB}}$ & $12.13 \pm 0.15^{\mathrm{A}}$ & $31.36 \pm 0.50^{\mathrm{A}}$ \\
Wheat flour + 15\% BW & $65.07 \pm 0.03^{\mathrm{A}}$ & $8.72 \pm 0.45^{\mathrm{C}}$ & $7.12 \pm 0.13^{\mathrm{A}}$ & $53.37 \pm 5.31^{\mathrm{B}}$ & $12.21 \pm 0.36^{\mathrm{A}}$ & $31.31 \pm 0.11^{\mathrm{A}}$ \\
\hline
\end{tabular}

Means \pm standard deviations $(n=3)$. Values in the same column with different letters differ significantly $(p<0.05)$

Table 3 Moisture and texture properties of dough incorporated with buckwheat flour

\begin{tabular}{lllll}
\hline Dough samples & Stickiness $(\mathrm{g})$ & Cohesiveness $(\mathrm{mm})$ & Extension $(\mathrm{g})$ & Extensibility $(\mathrm{mm})$ \\
\hline Control & $46.22 \pm 1.94^{\mathrm{C}}$ & $4.46 \pm 0.72^{\mathrm{A}}$ & $38.99 \pm 4.78^{\mathrm{A}}$ & $37.06 \pm 0.51^{\mathrm{A}}$ \\
Dough $+5 \% \mathrm{BW}$ & $51.76 \pm 5.25^{\mathrm{C}}$ & $2.32 \pm 0.45^{\mathrm{B}}$ & $35.72 \pm 2.42^{\mathrm{A}}$ & $36.14 \pm 0.46^{\mathrm{AB}}$ \\
Dough $+10 \% \mathrm{BW}$ & $62.01 \pm 5.72^{\mathrm{B}}$ & $1.35 \pm 0.87^{\mathrm{BC}}$ & $22.63 \pm 1.93^{\mathrm{B}}$ & $34.90 \pm 0.42^{\mathrm{B}}$ \\
Dough $+15 \% \mathrm{BW}$ & $77.59 \pm 5.39^{\mathrm{A}}$ & $0.76 \pm 0.36^{\mathrm{C}}$ & $17.54 \pm 1.24^{\mathrm{B}}$ & $34.60 \pm 0.21^{\mathrm{B}}$ \\
\hline
\end{tabular}

Means \pm standard deviations $(n=3)$. Values in the same column with different letters differ significantly $(p<0.05)$
Table 4 Physical properties of steamed bread

\begin{tabular}{llll}
\hline CSB samples & Loaf height $(\mathrm{mm})$ & Specific volume $(\mathrm{mL} / \mathrm{g})$ & Moisture $(\mathrm{g}$ water/100 g sample) \\
\hline Control & $62.14 \pm 0.38^{\mathrm{A}}$ & $2.47 \pm 0.03^{\mathrm{A}}$ & $40.10 \pm 0.01^{\mathrm{C}}$ \\
$\mathrm{CSB}+5 \% \mathrm{BW}$ & $59.65 \pm 0.14^{\mathrm{B}}$ & $2.36 \pm 0.03^{\mathrm{B}}$ & $40.15 \pm 0.02^{\mathrm{BC}}$ \\
$\mathrm{CSB}+10 \% \mathrm{BW}$ & $58.37 \pm 0.37^{\mathrm{C}}$ & $2.25 \pm 0.02^{\mathrm{C}}$ & $40.31 \pm 0.08^{\mathrm{B}}$ \\
$\mathrm{CSB}+15 \% \mathrm{BW}$ & $57.65 \pm 0.30^{\mathrm{C}}$ & $2.19 \pm 0.01^{\mathrm{D}}$ & $41.15 \pm 0.10^{\mathrm{A}}$ \\
\hline
\end{tabular}

Means \pm standard deviations $(n=3)$. Values in the same column with different letters differ significantly $(p<0.05)$

Table 5 Textural properties of steamed bread

\begin{tabular}{lllllll}
\hline Samples & Hardness $(\mathrm{g})$ & Springiness $(-)$ & Cohesiveness (-) & Gumminess (g) & Chewiness (g) & Resilience (-) \\
\hline Control & $228.24 \pm 25.92^{\mathrm{C}}$ & $0.94 \pm 0.01^{\mathrm{A}}$ & $0.88 \pm 0.01^{\mathrm{A}}$ & $191.75 \pm 19.15^{\mathrm{C}}$ & $179.83 \pm 19.34^{\mathrm{C}}$ & $0.57 \pm 0.01^{\mathrm{A}}$ \\
$\mathrm{CSB}+5 \% \mathrm{BW}$ & $287.95 \pm 19.01^{\mathrm{BC}}$ & $0.93 \pm 0.01^{\mathrm{AB}}$ & $0.87 \pm 0.02^{\mathrm{AB}}$ & $249.35 \pm 22.22^{\mathrm{BC}}$ & $229.44 \pm 24.05^{\mathrm{BC}}$ & $0.57 \pm 0.03^{\mathrm{A}}$ \\
$\mathrm{CSB}+10 \% \mathrm{BW}$ & $333.99 \pm 56.38^{\mathrm{AB}}$ & $0.91 \pm 0.01^{\mathrm{B}}$ & $0.86 \pm 0.01^{\mathrm{AB}}$ & $289.81 \pm 50.98^{\mathrm{AB}}$ & $244.82 \pm 41.47^{\mathrm{B}}$ & $0.54 \pm 0.02^{\mathrm{AB}}$ \\
$\mathrm{CSB}+15 \% \mathrm{BW}$ & $401.31 \pm 61.49^{\mathrm{A}}$ & $0.89 \pm 0.01^{\mathrm{C}}$ & $0.85 \pm 0.01^{\mathrm{B}}$ & $345.79 \pm 55.80^{\mathrm{A}}$ & $307.97 \pm 49.46^{\mathrm{A}}$ & $0.53 \pm 0.01^{\mathrm{B}}$ \\
\hline
\end{tabular}

Means \pm standard deviations $(n=3)$. Values in the same column with different letters differ significantly $(p<0.05)$

Table 6 Total starch and AUC of steamed bread

\begin{tabular}{lll}
\hline Samples & Total starch $(\%)$ & AUC \\
\hline Control & $43.82 \pm 1.30^{\mathrm{A}}$ & $431.51 \pm 31.80^{\mathrm{A}}$ \\
$\mathrm{CSB}+5 \%$ BW & $42.75 \pm 1.85^{\mathrm{A}}$ & $403.08 \pm 7.36^{\mathrm{A}}$ \\
$\mathrm{CSB}+10 \% \mathrm{BW}$ & $42.55 \pm 0.32^{\mathrm{A}}$ & $393.09 \pm 23.08^{\mathrm{AB}}$ \\
$\mathrm{CSB}+15 \% \mathrm{BW}$ & $42.07 \pm 0.21^{\mathrm{A}}$ & $337.27 \pm 21.11^{\mathrm{B}}$ \\
\hline
\end{tabular}

Means \pm standard deviations $(n=3)$. Values in the same column with different letters differ significantly $(p<0.05)$ 